\title{
Thrombosis of persistent median artery as a cause of carpal tunnel syndrome : case report
}

\author{
S. Arnauw, G. De Wachter
}

From the Department of orthopaedic surgery, Jessa Hospital, 3500 Hasselt, Belgium

\begin{abstract}
Carpal tunnel syndrome (CTS) is a common peripheral neuropathy, caused by compression of the median nerve. Symptoms usually are present for months and aggravate over time. Acute onset of complaints and symptoms, like coldness of the hand, should raise awareness of a possible vascular cause of CTS.Persistent median artery (PMA) is a very rare anatomical variant of the blood supply of the upper limb. The presence of a thrombosed PMA is an extremely rare cause of CTS. In this article a case is presented in which the patient has carpal tunnel syndrome of his left hand, caused by a thrombosed persistent median artery.

Conservative treatment, consisting of rest, ice application and non-steroidal anti-inflammatory drugs, failed. Surgical excision of the thrombosis and open exploration of the carpal tunnel was performed, with complete relief of symptoms. In literature different treatment options, like conservative treatment with antiplatelet therapy or surgical excision of the thrombosis and decompression of the nerve, are described with good results. However up until now, no consensus exists about the golden standard in treatment of a thrombosed persistent median artery.
\end{abstract}

Key words : carpal tunnel syndrome ; persistent median artery ; thrombosis.

No benefits or funds were received in support of this study. None of the authors have a conflict of interest.

\section{INTRODUCTION}

Carpal tunnel syndrome is a frequently seen peripheral neuropathy, caused by compression of the median nerve. Common symptoms are numbness, paresthesia and pain in the first three fingers (ie. nerve territory of the median nerve). Usually, these complaints have been present for a long time and aggravated progressively (4-8).

The median artery is part of the embryological arterial axis of the upper limb. Together with the anterior interosseus artery, it's the main source of blood supply during the first trimester of pregnancy. Normally, the median artery regresses around 8 weeks after gestation (41-4).

Many vascular anomalies of the blood supply of the wrist and hand region have been documented, with the very rare presence of a persistent median artery (PMA) being one of them $(1,2)$. PMA is often accompanied by a bifid median nerve, an anatomical variation with a division of the median nerve proximal of the carpal tunnel.

Sebastiaan Arnauw ${ }^{1}, \mathrm{MD}$,

Gerrit De Wachter ${ }^{2}$, MD

${ }^{1}$ Resident at Department of orthopaedic surgery, Jessa Hospital, 3500 Hasselt, Belgium.

${ }^{2}$ Staff surgeon at Department of orthopaedic surgery, Jessa Hospital, 3500 Hasselt, Belgium.

Correspondence : Gerrit De Wachter, Brugstraat 83, 3500

Hasselt, Belgium. Phone : 0473918297.

Email : gerritdewachter@hotmail.com

- 2021, Acta Orthopædica Belgica. 


\section{CASE REPORT}

A 39-year-old male was referred to our department of Orthopedic Surgery after presenting at the emergency department with atraumatic pain and paresthesia in both wrists, carpal and metacarpal regions, with the dominant left wrist affected the most. Symptoms started a few weeks before, increased progressively and are described as a cramping, tingling feeling and a burning pain that is worse at night.

Tender swelling on the volar side of the left wrist was observed. There was no thenar or hypothenar atrophy present. Palpation of the left wrist was painful. No abnormalities during active and passive range of motion of both wrists and hands were seen. Bilateral Tinel's sign and Phalen's maneuver were executed and for both tests a positive result of the left hand was noted. Blood supply of the hand was assessed by Allen's test, with a negative test result bilateral.

Radiography of both wrists and hands showed no deformities.

Ultrasonography of the left wrist and hand was performed and showed a thrombosis of a persistent median artery, starting proximally of the carpal tunnel going more distally, with a length of $3 \mathrm{~cm}$. Maximum diameter of the thrombosis was $3 \mathrm{~mm}$. At the proximal side of the thrombosis, a small network of collateral blood vessels was visible. Preoperative Doppler examination was not performed.

(Venous blood analysis during admittance on the emergency room showed no particularities whatsoever: CRP $<0.30 \mathrm{mg} / \mathrm{L}$, leukocytes 6.37, rheumatoid factor $<10 \mathrm{IU} / \mathrm{L}$.

Electromyography showed no signs of peripheral neurogenic or myogenic suffering)

Our first treatment of choice was conservative, consisting of non-steroidal anti-inflammatory drugs and ice application. Unfortunately, this conservative treatment failed. After explaining different treatment procedures, possible complications and outcomes, the patient agreed to have surgical treatment. Open carpal tunnel release was performed while using a tourniquet. First, the point of incision was marked using the intersection between the Kaplan cardinal line and the radial side of the fourth ray as a

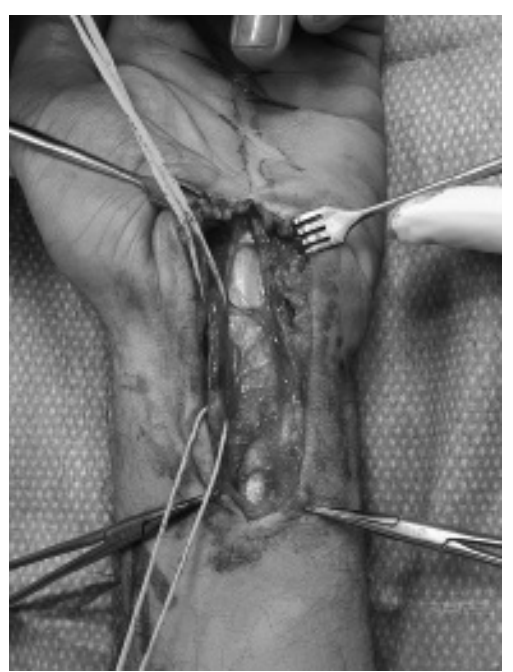

Fig. 1. - Exploration of the carpal tunnel with vessel loops around the thrombosed persistent median artery. (The median nerve is enlarged, but shows no abnormalities).

landmark. From this point, an incision of about $4 \mathrm{~cm}$ in proximal direction was made. Next, superficial dissection was carried out, until the transverse carpal ligament was visible. This ligament was then carefully released and the thrombosed persistent median artery (PMA) was seen inside the carpal tunnel. After exploring and dissecting it from the median nerve and soft tissue, the PMA was clipped on both sides of the thrombosis and the thrombosis was extracted. Exploration of the median nerve showed no abnormalities, there was no bifid median nerve. Tourniquet was taken off to control hemostasis. The wound was rinsed with saline, and then closed by using Ethilon 3/0 sutures.

No postoperative complications were reported. Sutures were removed two weeks after surgery. At one-month follow-up, complaints had completely disappeared and a normal functionality of the wrist and hand was obtained. Duplex ultrasonography was performed to evaluate blood supply of the hand, with a normal flow of the ulnar and radial artery and vein. Three-month follow-up was scheduled, but had to be postponed due to the Covid-19 pandemic.

\section{DISCUSSION}

Carpal tunnel syndrome is a common peripheral neuropathy, caused by compression of the median 
nerve. Numerous pathologies, such as diabetes mellitus, hypothyroidism, rheumatoid arthritis or wrist fractures, can cause CTS (4-10). Usually, symptoms which include numbness of the first three digits, paresthesia and nocturnal pain, are present for months and deteriorate over time. Acute onset of symptoms, including coldness of the hand, and absence of trauma have to raise suspicion of a vascular cause of CTS (6).

The median artery is part of the arterial axis of the upper limb during the first stages of ontogeny. It has an important role in blood supply of the hand in the first months of embryologic development and normally regresses in the second month after gestation (1-3).

Persistent median artery (PMA) is an anatomical variation that occurs when the median artery fails to regress. Prevalence of PMA varies widely, with different studies describing ranges from $1.1 \%$ to $16.1 \%$ (2) or even $0.6 \%$ to $21.1 \%$ (1). No significant difference between groups (sex, age, dominant hand) is reported (3). Different types of PMA are described : an antebrachial type (which doesn't play a role in blood supply of the hand) and a palmar type. The palmar type PMA contributes to the formation of the superficial palmar arch, together with the ulnar artery. PMA, with diameter $1-1.5 \mathrm{~mm}$, is smaller than the ulnar and radial nerve (1-2).

The presence of PMA is often accompanied by the presence of bifid median nerve, an anatomical variation of the median nerve. Bifid median nerve means that the nerve is divided proximal to the entry of the carpal tunnel. However, in our case we found a normal anatomy of the median by surgical exploration. Bifid median nerve and asymptomatic PMA with normal diameter also occur in healthy people and don't pose a risk itself for developing CTS (1,2,3,6,9-11).

Nevertheless, symptomatic PMA (i.e. diameter of at least $3 \mathrm{~mm}$, or atherosclerosis, or internal thrombus) is observed to be an additional risk factor for CTS, by causing compression of the median nerve. Thrombosed persistent median artery can be drug induced or it can be the result of trauma or overuse of the hand (4-7).

Ultrasonography of the wrist and Doppler examination with a high frequency probe are reported to be ideal imaging tools for diagnosing PMA, with several case reports stating that CT or MRI could be useful to evaluate the anatomy of the wrist (3-8).

Conservative treatment, consisting of drugs (i.e. analgesia, aspirin and statin), is described in most studies and case reports, with satisfying results. Surgical treatment by removing the thrombosis, as in our case, is reported to be effective, but up until now, no consensus about the treatment of a thrombosed PMA has been reached (4-8).

\section{CONCLUSION}

Acute carpal tunnel syndrome is not often seen, and should raise suspicion of a vascular cause. CTS, caused by thrombosis of persistent median artery, is a rare pathology, with no golden standard in diagnostic and therapeutic measures at this moment.

Knowledge about anatomic variations is critical for surgeons, to prevent intra- and postoperative complications (e.g. bleeding).

\section{REFERENCES}

1. Haladaj R, Wysiadecki G, Dudkiewicz Z, Polguj M, Topol M. Persistent Median Artery as an Unusual Finding in the Carpal Tunnel : Its Contribution to the Blood Supply of the Hand and Clinical Significance. Med Sci Monit. 2019 January ; 25 : 32-39.

2. Singla RK, Kaur N, Dhiraj GS. Prevalence of the Persistent Median Artery. Journal of Clinical and Diagnostic Research. 2012 November ; 6(9) : 1454-1457.

3. Chen L, Chen J, Hu B, Jiang LX. Sonographic Findings of the Bifid Median Nerve and Persistent Median Artery in Carpal Tunnel : A Preliminary Study in Chinese Individuals. Clinics. 2017 ; 72(6) : 358-362.

4. Feintisch AM, Haripriya SA, Datiashvili R. An Anatomic Variant of Persistent Median Artery in Association with Carpal Tunnel Syndrome : Case Report and Review of the Literature. J Hand Surg Asian-Pac. 2017 ; 22(4) : 523-525

5. Butt J, Ahluwalia AK, Dutta A. Incidental finding of a persistent median artery (palmar type) during a routine carpal tunnel decompression : a case report. Ann $R$ Coll Surg Engl. 2017 ; 99 : e204-e205

6. Akgun AS, Ertan G, Ulus S. Acute carpal tunnel syndrome caused by thrombosed persistent median artery associated with bifurcated median nerve in a pregnant woman. BMJ Case Rep [Internet]. 2017 September 19 [cited 2020 May]. Available from https://casereports.bmj.com/content/2017/ bcr-2017-221446. DOI : 10.1136/bcr-2017-221446. 
7. Avenel M, Miranda S, Benhamou Y, Michelin P, Boyer JC, Lévesque $\mathbf{H}$, et al. Canal carpien aigu : penser à la thrombose de l'artère persistante du nerf median. La Revue de médecine interne. 2019 ; $40:$ 453-456.

8. Rzepecka-Wejs L, Multan A, Konarzewska A. Thrombosis of the persistent median artery as a cause of carpal tunnel syndrome - case study. J Ultrason. 2012 ; 12 : 487-492.

9. Trachani E, Rigopoulou A, Veltsista D, Gavanozi E, Chrysanthopoulou A, et al. Occurrence of bifid median nerve in healthy and carpal tunnel syndrome patients. Journal of Electromyography and Kinesiology. 2018 ; 39 : 77-80.

10. Kostoris F, Bassini S, Longo E, Murena L. Carpal Tunnel Syndrome Associated with Bifid Median Nerve and Palmaris Profundus - Case Report and Literature Review. $J$ Hand Surg Asian-Pac. 2019 ; 24(2) :238-242.

11. Lanz U. Anatomical variations of the median nerve in the carpal tunnel. J Hand Surg Am. 1977 ;2(1) :44-53. 